\title{
Direct Determination of Medium Range Ordering in Amorphous Hydrogenated Boron Carbide for Low-k Dielectric Applications
}

Mehrdad Abbasi Gharacheh ${ }^{1}$, Soohyun Im $^{1}$, Jared Johnson ${ }^{1}$, Gabriel Calderon Ortiz ${ }^{1}$, Menglin Zhu ${ }^{1}$, Nathan Oyler ${ }^{2}$, Michelle Paquette ${ }^{2}$, Paul Rulis ${ }^{2}$, Ridwan Sakidja ${ }^{3}$ and Jinwoo Hwang ${ }^{1}$

${ }^{1}$ The Ohio State University, Columbus, Ohio, United States, ${ }^{2}$ University of Missouri - Kansas City, Kansas City, Missouri, United States, ${ }^{3}$ Missouri State University, Springfield, Missouri, United States

During past decades, the frequency of micro-processors has increased from $66 \mathrm{MHz}$ to $4 \mathrm{GHz}$ while their sizes have decreased from $1 \mu \mathrm{m}$ to $90 \mathrm{~nm}$. As the size of interconnections (Fig. 1a) reduces, the conductors' resistance and insulators' capacitance increase, leading to increase in Resistance-Capacitance (RC) delay of ICs. It is important to decrease either conductors' resistance or dielectrics' capacitance to have more densely integrated transsitors and less RC delay [1]. To achieve this, there have been ongoing efforts to substitute traditional $\mathrm{SiO}_{2}$ with new generation of low dielectric constant (k) materials. Simultaneously, it is crucial to have stability in mechanical and thermal properties along with low dielectric constant [23].

Amorphous hydrogenated boron carbide $(\mathrm{a}-\mathrm{BC}: \mathrm{H})$ is a desired option containing both proper mechanical properties and low dielectric constant. The a-BC:H films can be grown using plasma enhanced chemical vapor deposition (PECVD) with ortho-carborane precursors [4], which results in a wide range of variation in the structure and composition. Theoretical modeling of amorphous hydrogenated boron carbide has suggested that atomic structure of a-BC:H contains 12-vertex icosahedral clusters which are attributed to short range ordering (SRO, Fig. 2b) [5]. These SRO clusters may gather and form the medium range ordering (MRO). However, it is currently not clear whether MRO information from these simulations is accurate since some simulation parameters (such as the time scale) are substantially different from the real growth conditions. The MRO parameters, if properly characterized, will provide us the opportunity to control the properties of the amorphous films by precisely controlling the PECVD growth conditions. However, MRO characterization has been challenging because conventional imaging or diffraction techniques tend to be insensitive to MRO.

In this report, we characterize the size of MROs in real space as a function of the scattering vector, $k$, based on 4-dimensional scanning transmission electron microscopy (4D-STEM). Using 4D-STEM, we acquired thousands of 2D nano-difraction from 2D areas of the amorphous samples (Fig. 2a). The scattered electrons from the sample are counted by an electron microscopy pixel array detector (EMPAD, Fig. 1c), which has high dynamic range (32-bit) as well as fast data acquisition rate. The layers of nano-diffraction patterns then are reconstructed as a function of $k$ and $\varphi$ as shown in Fig. 2a and 2b. $k$ has the increment of 1 pixel and $\varphi$ has the increment of $0.05 \mathrm{rad}$. Speckles show up as pixels with higher intensities in realspace reconstructed images, which are directly attributed to MROs. Mapping of particles is possible based on the analysis of intensity histograms fitted with normal distribution (Fig. 2c), which provides a threshold for particles recognition in each reconstructed image with one specific $k$ and $\varphi$. Hence, particles' sizes in real space are determined over entire $k$ range. 2D histogram of distribution of particles sizes vs $\mathrm{k}$ vector (Fig. 2d), clearly reveals that MROs have a range 3-6nm in diameter. These results can be later combined with symmetry results of MROs to provide a better description for relating the overall property to medium range ordering in amorphous hydrogenated boron carbides and other disordered systems. 
(a)

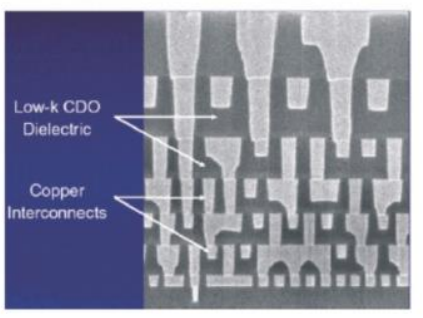

(b)

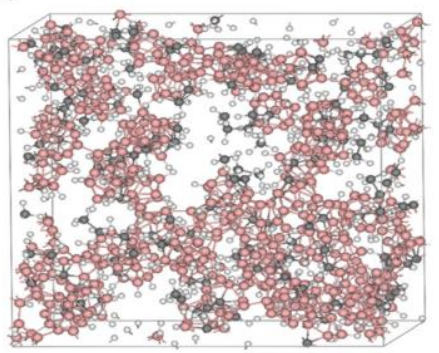

(c)

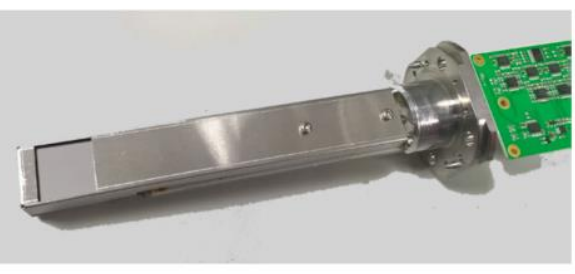

Figure 1. Figure 1. (a) structure of an interconnect [1], (b) an atomic model of a-BC:H [4], and (c) EMPAD.

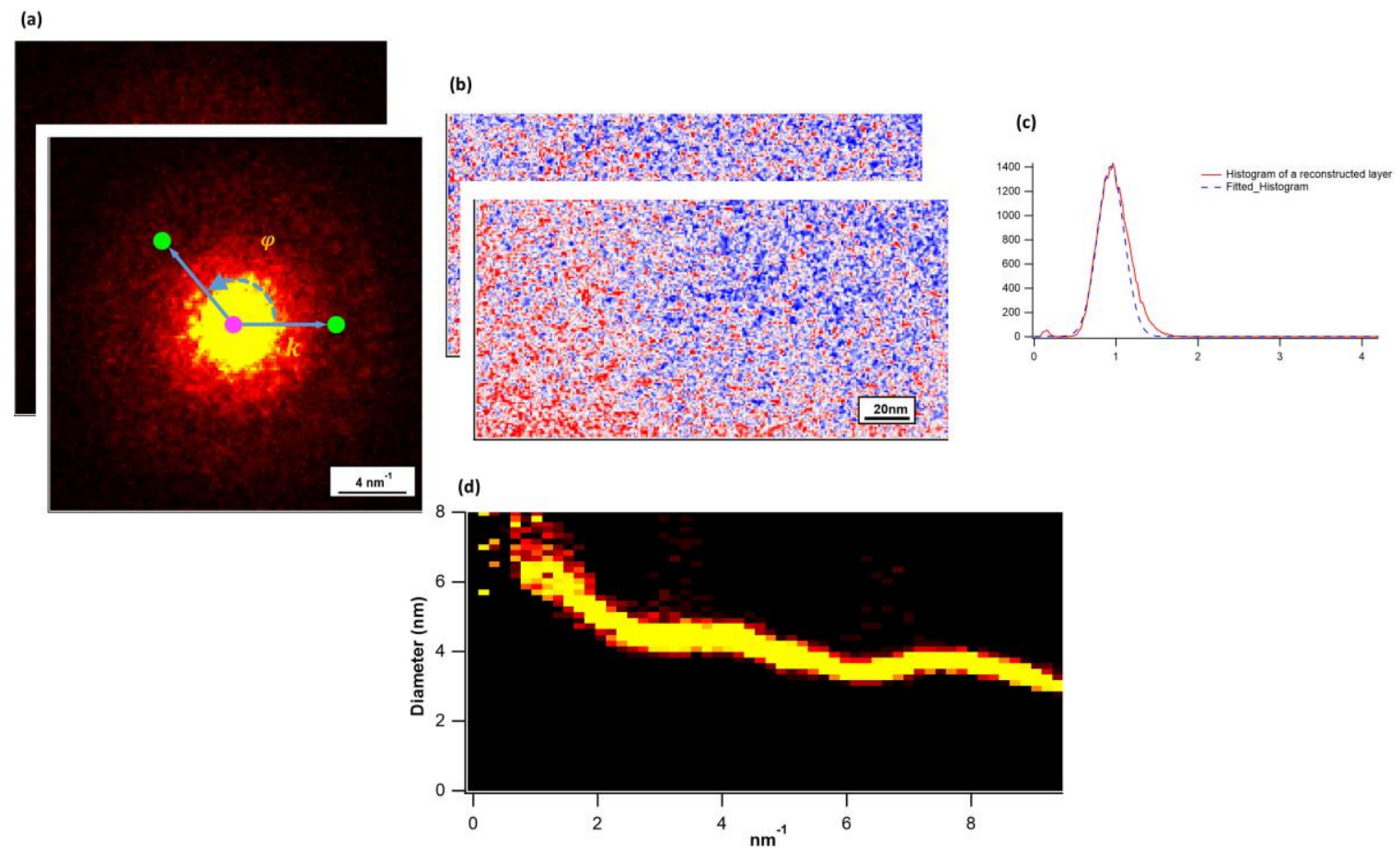

Figure 2. Figure 2. (a) layers of a-BC:H nano-diffraction patterns, (b) reconstructed images of nanodiffractions at one $\mathrm{k}$ and $\varphi$ with visible speckles, (c) a typical histogram of intensities in a reconstructed image, and (d) a 2D histogram of distribution of MROs sizes as a function k vector.

\section{References}

[1] M. R. Baklanov, P. S. Ho, and E. Zschech, Advanced Interconnects for ULSI Technology. 2012.

[2] D. Shamiryan, T. Abell, F. Iacopi, and K. Maex, "Low-k dielectric materials," no. January, pp. 3439, 2004.

[3] A. Grill, S. M. Gates, T. E. Ryan, S. V. Nguyen, and D. Priyadarshini, "Progress in the development and understanding of advanced low $\mathrm{k}$ and ultralow $\mathrm{k}$ dielectrics for very large-scale integrated interconnects - State of the art," Appl. Phys. Rev., vol. 1, no. 1, 2014.

[4] B. J. Nordell et al., "The influence of hydrogen on the chemical, mechanical, optical/electronic, and electrical transport properties of amorphous hydrogenated boron carbide," J. Appl. Phys., vol. 118, no. 3, 2015.

[5] D. Emin, "Unusual properties of icosahedral boron-rich solids," J. Solid State Chem., vol. 179, no. 9, pp. 2791-2798, 2006. 